\title{
Regionale Sprache als Identitätsmarker Hamburger Fußballfans. Eine Projektskizze
}

\author{
Lara Neumann (Hamburg)
}

\begin{abstract}
The dissertation project Regionale Sprache als Identitätsmarker. Zur Konstruktion sozialer Bedeutung bei Hamburger Fußballfans 'Regional language as identity marker. The construction of social meaning among football fans in Hamburg' examines different practices of identity construction of fans from the two football clubs FC St. Pauli and Hamburger Sportverein (HSV). By analysing a group interview with HSV fans, this paper investigates the potential of identification with regional language. Identity constructions can be identified in the following three aspects: (a) the conceptualisation of the local substandard, (b) the speakers' positioning concerning the use of the local substandard and (c) language attitudes.
\end{abstract}

\section{$1 \quad$ Einleitung}

„Beim Fußball [...] da sprichst du halt, wie's grade vonne Zunge rollt.“ Mit diesen Worten beschreibt GP03 (männlich, *1983) seinen Sprachgebrauch als Zuschauer bei Spielen seines favorisierten Fußballvereins, dem Hamburger Sportverein (HSV). Die ungezwungene Kommunikation im sportlichen Rahmen wird in dieser Äußerung nicht nur thematisiert, sondern durch die standardabweichende Realisierung des Gesagten auch sprachlich hervorgebracht. Das Zitat stammt aus einem Gruppeninterview mit HSV-Fans, das im Rahmen des hier vorzustellenden Dissertationsvorhabens mit dem Arbeitstitel „Regionale Sprache als Identitätsmarker. Zur Konstruktion sozialer Bedeutung bei Hamburger Fußballfans“" durchgeführt wurde. ${ }^{1}$

Im Projekt werden verschiedene Praktiken der sprachlichen wie außersprachlichen Identitätskonstruktion am Beispiel von Hamburger Fußballfans untersucht. Fußballfans stehen eine Vielzahl von Mitteln zur Verfügung, um ihre soziale Zugehörigkeit zu markieren und um sich

\footnotetext{
${ }^{1}$ In Anlehnung an Schmidt/Herrgen (2011: 15) wird im Dissertationsprojekt unter dem Terminus ,regionale Sprache“ der Sprechlagenbereich verstanden, der sich „,unterhalb“ der gesprochenen Standardsprache“ befindet bis einschließlich hin zum (hamburgischen) Niederdeutsch. Die Bezeichnungen „Hamburgisch“ und „Substandard“ werden in diesem Beitrag für den mittleren Sprechlagenbereich, in Abgrenzung zum genuin „Niederdeutschen“, synonym verwendet.

Der Begriff ,(Identitäts-)Marker“ bezieht sich auf die verfügbaren Zeichen, deren Gebrauch soziale Zugehörigkeit (kontextspezifisch) zum Ausdruck bringt (vgl. hierzu grundlegend Labov 1972; anhand niederdeutscher Beispiele vgl. Elmentaler/Niebuhr 2017: 109-111). Steht dagegen der identitätsstiftende Aspekt des Zeichengebrauchs im Vordergrund, wird der Terminus ,,(Identitäts-)Konstruktion“ vorgezogen. Dieser schließt zugleich noch weitere zu analysierende Faktoren (z. B. Einstellungen) mit ein, die aktiv an der Identitätsbildung beteiligt sind (zur theoretischen Modellierung von Identitätskonstruktionen vgl. Schröder 2019; zum Identitätsbegriff vgl. Kap. 3.1).

Linguistik online 99, 6/19 - http://dx.doi.org/10.13092/lo.99.5968
}

CC by 3.0 
zugleich von den Fans anderer Vereine abzugrenzen. ${ }^{2}$ Während eines Fußballspiels sind dies die Fans des Gegners; außerhalb des Stadions, also im Alltag der Fans, sind es dagegen oft die Fans eines anderen regional oder lokal agierenden Vereins. So ist es auch in Hamburg, wo der FC St. Pauli und der HSV in unmittelbarer Konkurrenz zueinander stehen. ${ }^{3}$ Aufgrund der großen Bedeutung von Rivalität - insbesondere lokaler - für die Identitätsbildung (vgl. Winands/Grau/Zick 2017: 8) befasst sich das Dissertationsprojekt mit den Fans der beiden größten Hamburger Fußballvereine. ${ }^{4}$

Neben nonverbalen Mitteln der Identitätsmarkierung, wie bspw. Bekleidung, nimmt das Projekt auch den regionalen Sprachgebrauch der in Hamburg lebenden Fans in den Blick. Damit schließt das Vorhaben an gegenwärtige variationslinguistische Untersuchungen an, die sprachliche Variation in einem erweiterten semiotischen Kontext betrachten (zur Entwicklung der variationslinguistischen Forschung vgl. Eckert 2012) und zugrunde legen, dass sprachlichen Zeichen eine soziale Bedeutung zukommt, indem Sprecher*innen durch den Sprachgebrauch ihre Identität (mit-)konstituieren können (vgl. Johnstone 2010: 31).

In Bezug auf regionale Sprachformen in Hamburg wurde bereits festgestellt, dass „Niederdeutsch und Missingsch ein fester Bestandteil des Selbstverständnisses der Hamburger Bürger“ (Elmentaler/Niebuhr 2017: 135; vgl. ebenso Jürgens 2016) geworden sind. Darüber hinaus hat sich im Zuge von Analysen zur Sprachwahrnehmung und zu Spracheinstellungen in Hamburg herauskristallisiert, dass zwischen der Identitätsbildung von Hamburger Fußballfans und regionalem Sprachgebrauch ein Zusammenhang angenommen werden kann (vgl. Schröder 2015: 50f.). Als Indiz für eine sprachliche Identitätsstiftung können nicht zuletzt die durch die Fans selbstgewählten Fanclubnamen gewertet werden: So gehören zu den mit einer Hamburger Postleitzahl registrierten HSV-Fanclubs u. a. die „Hamborger Kinners“, „Rop un rünner“ oder die „Elbjungs \& Elbdeerns“ (HSV Fußball AG s. a.); zu den lokalen FC St. Pauli Fanclubs zählen „Mein Viddel, meine Loide, mein Verein“, „Wi Köönt Ok Anners“ oder „FC St.Pauli dör un dör“ (Fanclubsprecherrat St. Pauli 2018).

Der vorliegende Beitrag soll einen Einblick in das Dissertationsprojekt geben. Zunächst werden die Untersuchungsziele und Fragestellungen vorgestellt und die zugrunde liegenden theoretischen Konzepte dargelegt, zu denen der Identitätsbegriff, die Einstellung und die Positionierung gehören. Im Anschluss daran werden das Design und das methodische Vorgehen erläutert. Angelehnt an das eingangs angeführte Zitat wird im Zentrum dieses Beitrags ein Gespräch stehen, das mit einer Gruppe von HSV-Fans geführt wurde. Die Analyse ausgewählter Interviewausschnitte wird zeigen, auf welche Weise regionale Sprache von den Fußballfans konzeptualisiert und bewertet sowie zur Identitätskonstruktion eingesetzt wird.

\footnotetext{
${ }^{2}$ Von der Heyde/Kotthaus/Weitzel (2018: 148) verweisen auf die regionale Zugehörigkeit, die ,im Gebrauch von Artefakten wie Trikots, Kaffeetassen oder Autoaufklebern“ ausgedrückt wird.

${ }^{3}$ Die beiden Vereine spielen nach dem Abstieg des HSV in die 2. Bundesliga in der Saison 2018/2019 erstmals seit der Bundesligasaison 2010/2011 gegeneinander, sodass die lokale Rivalität im Zeitraum der Datenerhebung zusätzlich geschürt wird. Zur Lokalrivalität sowie zu Unterschieden in den Fanlagern vgl. Verein Jugend und Sport e.V. (1996: 7-11).

${ }^{4}$ Es wurden bewusst Vereine ausgewählt, die denselben räumlichen und regionalsprachlichen Bezugspunkt aufweisen (vgl. Kap. 2), wenngleich eine Gegenüberstellung von Fans anderer (rivalisierender) Vereine ebenso denkbar wäre, z. B. zwischen dem HSV und dem SV Werder Bremen oder dem FC St. Pauli und dem F.C. Hansa Rostock.
} 


\section{Untersuchungsziele und Fragestellungen}

Das Ziel des Dissertationsprojekts ist es, den Zusammenhang zwischen regionalen Sprachformen und Identitätskonstruktionen von Hamburger Fußballfans zu ergründen. ${ }^{5}$ Den Ausgangspunkt bildet dabei, dass Personen und soziale Gruppen mithilfe verschiedener Praktiken, d. h. dem Rückgriff auf sprachliche und außersprachliche Mittel und Verhaltensweisen, soziale Zugehörigkeit konstituieren können. Einzelne (sprachliche) Merkmale stehen also nicht per se mit bestimmten Identitäten im Zusammenhang, sondern entfalten im Wechselspiel mit weiteren Komponenten ihre soziale Bedeutung (vgl. Eckert 2008: 456; zum Identitätsbegriff vgl. Kap. $3.1)$.

Das Projekt untersucht zum einen für einzelne Fans bzw. Fangruppen die semiotischen Mittel, welche soziale Zugehörigkeit anzeigen, wie z. B. sichtbare Symbole auf der Kleidung oder auf Alltagsgegenständen. Zum anderen werden Verhaltensweisen behandelt, die das doing-fan (vgl. Grau/Winands 2017: 66) betreffen und das Selbstbild der Fans oder Fangruppen mitkonstituieren (vgl. Kap. 4). Aufgrund des Einflusses von Rivalität auf die Identitätsbildung von Fußballfans sollen Abgrenzungsmechanismen aufgedeckt werden, die sowohl gegenüber Fans des Lokalrivalen ${ }^{6}$ als auch gegenüber anderen Fangruppen des eigenen Vereins auftreten können. ${ }^{7}$ Dabei sollen die Einstellungen und Positionierungen der Fußballfans analysiert werden sowie die sprachlichen Mittel, die sie hervorbringen.

Durch eine Analyse des Sprachgebrauchs ${ }^{8}$ soll festgestellt werden, welche Funktion regionale Sprache innerhalb eines umfassenderen Symbolsystems erfüllt. Es wird überprüft, auf welche Identitätskonzepte regionale Sprachmerkmale rekurrieren, genauer gesagt, welche verwendeten Varianten (verschiedene) soziale Bedeutungen generieren, insbesondere vor dem Hintergrund, dass beide Vereine in derselben Stadt agieren. Um das Identifikationspotential regionaler Sprachformen zu ermitteln, werden einerseits Wissensbestände und Spracheinstellungen betrachtet sowie die vorgenommenen Positionierungen, die die Einstellung im Gespräch zum

\footnotetext{
${ }^{5}$ Grau/Winands (2017: 66) weisen darauf hin, dass die Präzisierung des Fanbegriffs ,je nach interessierender Forschungsfrage unterschiedlich festzulegen" sei. Die Gewährspersonen des Dissertationsprojekts kategorisieren sich selbst durch die Studienteilnahme als (echte) Fans ihres Vereins. Diese Selbstkategorisierung wird als Anknüpfungspunkt genutzt, um die Gewährspersonen den Fanbegriff im Rahmen der Studie eigenständig modellieren zu lassen. Zur Auswahl der Gewährspersonen und zum Studiendesign vgl. Kap. 4.

${ }^{6}$ Als Ursache für die Rivalität zwischen den Fanlagern des FC St. Pauli und des HSV nennt der Verein Jugend und Sport e.V. (1996: 11) u. a. ,die unterschiedlichen (Fan-)Lebensgeschichten, de[n] unterschiedliche[n] Grad der Identifikation mit dem Verein und die z.T. unterschiedlichen Motive für das jeweilige Fandasein bzw. die Spielbesuche" sowie (vorurteilsbehaftete) Zuschreibungen, welche die politische Orientierung betreffen.

${ }^{7}$ In der sozialwissenschaftlichen Fußballfanforschung wurden bereits verschiedene Fantypen klassifiziert, die hierbei von Bedeutung sein können (vgl. grundlegend Heitmeyer/Peter 1988: 32, welche die jugendliche deutsche Fanszenerie in konsumorientierte, fußballzentrierte und erlebnisorientierte Fans einteilen; Grau et al. (2016: 2728) differenzieren auf Basis einer quantitativen Erhebung fünf Fan-Kategorien, die sich in ihrer Konfliktbereitschaft unterscheiden (,security-oriented passive“, ,peaceful, supportive average“, „less aggressive supporters“, "active, emotional supporters" sowie ,active, confrontational conflict-seekers")).

${ }^{8}$ Dabei kann es sich sowohl um eine produktive Varietätenkompetenz als auch um Zitationen sprachlicher Stereotype oder um verwendete schriftliche Manifestationen (z. B. auf Fahnen oder T-Shirts) handeln. Welche Sprachvarianten hierbei eine Rolle spielen könnten, kann an dieser Stelle nicht weiter ausgeführt werden. Bei dieser Frage sind auch Faktoren wie sprachliche Wissensbestände oder die Salienz eines Merkmals miteinzubeziehen, vgl. hierzu Hettler (2018).
} 
Ausdruck bringen. Andererseits sollen objektsprachliche Analysen sichtbar machen, auf welche Weise regionale Sprachvarianten zur Identitätskonstruktion eingesetzt werden.

Dialektales Sprachverhalten kann mit positiven Einstellungen zum Wohnort korrelieren; als Indikator für Ortsloyalität kann wiederum die Mitgliedschaft in Vereinen gelten (vgl. Leuenberger 2000: 165-170). Das Projekt geht der Frage nach, ob dies gleichermaßen auf Fußballfans zutrifft, die in derselben Stadt leben, in der ihr Verein verankert ist. Dabei haben Untersuchungen im Ruhrgebiet bereits gezeigt, dass Profifußball ein wichtiges Mittel lokaler Identitätsstiftung darstellt (vgl. Gehrmann 1994: 200). Es werden daher stadtbezogene Zuschreibungen sowie Ortseinstellungen erhoben, welche Rückschlüsse auf zugrunde liegende regionale (und regionalsprachliche) Identitätskonzepte der einzelnen Fangruppen erlauben. In diesem Zusammenhang ist auch zu beleuchten, ob sich Unterschiede aus dem Umstand ergeben, dass es sich beim HSV um einen Stadt- und beim FC St. Pauli um einen Stadtteilverein handelt.

\section{Theoretische und methodische Grundlagen}

\subsection{Identität}

Der Begriff Identität greift gemeinhin zwei Aspekte auf: Zum einen ist Identität subjektgerichtet, indem sie das „,(innerliche) Selbstgefühl und Selbstbild“ einer Person, ihr eigenes „Selbstund Weltverhältnis“ (Rosa 2007: 47) beschreibt (personale Identität). ${ }^{9}$ Zum anderen konstituiert sich Identität durch die Zugehörigkeit zu sozialen Gruppen. Diese Dimension von Identität wird nach Taifel (1981: 255) als ,soziale Identität“ bezeichnet: „Social Identity will be understood as that part of an individual's self-concept which derives from his knowledge of his membership of a social group (or groups) together with the value and emotional significance attached to that membership. “10

Beide Ausprägungen werden in spezifischen Teilidentitäten sichtbar, z. B. in Form einer regionalen Identität, die sich ,aus den Vorstellungen und Einschätzungen über die eigene Region als Wahrnehmungsgegenstand und als Raum sozialer Beziehungen“ (Lilli 1994: 93) speist. Die Region stellt also nicht nur eine geographisch abgrenzbare Fläche dar; sie ist außerdem als ein historisches, ökonomisches, politisches, soziales und kulturelles Gebilde aufzufassen (vgl. Blotevogel 2001: 3).

Vor allem bezogen auf kulturelle Aspekte der regionalen Identität gilt Sprache bislang als essentielles Mittel der Identitätsbildung und -wahrung (vgl. Gleber 1994: 10; ähnlich Löffler 1998: 78). Unter dem Begriff „Sprachidentität“ wird „die Identität einer Person in Bezug auf ihre - oder auf eine - Sprache“ (Thim-Mabrey 2003: 2) verstanden. Zusätzlich kann Sprache aber auch aktiv zur Identitätskonstruktion genutzt werden. Analog zu den verschiedenen sozialen Teilidentitäten einer Person schlägt Kresić (2007: 15) ein Modell zur multiplen Sprachidentität vor, wonach eine Person mithilfe ihres Sprachgebrauchs kontextbezogen Teil-

\footnotetext{
9 Identität wird nicht als „starres Konzept“ aufgefasst, sondern hat stets „,die Mannigfaltigkeit menschlicher Interaktion im Ablauf von Biographien und angesichts sozialen Wandels“ (Krappmann 2004: 405) zu berücksichtigen. So werden Differenzerfahrungen oder Brüche in das Selbstbild integriert; sie können sogar als konstitutiv angesehen werden (vgl. Straub 2011: 285).

${ }^{10}$ Lilli (1994: 93) betont, dass lediglich einzelne soziale Teilidentitäten einer Person, und nicht die soziale Identität einer Person als Ganzes, zugänglich seien.
}

ISSN 1615-3014 
identitäten konstruieren könne, bspw. als Mutter oder Fußballfan (Kresić 2007: 16). Dieser Ansatzpunkt ist von Bedeutung für das Dissertationsprojekt, da die soziale Teilidentität als Fußballfan im Zentrum der Analyse steht. Untersucht wird allerdings nicht eine spezifische Sprache des Fußballs (vgl. hierzu zusammenfassend Burkhardt 2006: 7-11), sondern auf welche Weise regionale Sprache in die Identitätskonzepte Hamburger Fußballfans eingebunden wird. Den realisierten Sprachvarianten wird keine statische Bedeutung zugeschrieben (vgl. Eckert 2008: 453), vielmehr wird von einer indexikalischen Beziehung zwischen Identitätskonzepten und sprachlichen Merkmalen ausgegangen. ${ }^{11}$ Sprachliche Variation wird als Komponente eines spezifischen Stils interpretiert: „Persona style is the best level for approaching the meaning of variation, for it is at this level that we connect linguistic styles with other stylistic systems such as clothing and other commoditized signs" (Eckert 2008: 456). Demzufolge wird Identität im Dissertationsprojekt auf einen bestimmten (Gesprächs-)Kontext bezogen; Identität ist nicht direkt zugänglich, sondern spiegelt sich in stilistischen Praktiken (vgl. Eckert 2012: 94). ${ }^{12}$

\subsection{Einstellung}

Wie auch bei der Identität handelt es sich bei der Einstellung um ein ursprünglich sozialpsychologisches Konzept. Eine Einstellung ist stets auf ein bestimmtes Objekt gerichtet, wobei grundsätzlich jeder wahrnehmbare oder denkbare Gegenstand, konkret oder abstrakt, belebt wie unbelebt, als Einstellungsobjekt fungieren kann (vgl. Bohner 2002: 267 angelehnt an Eagly/Chaiken 1998 sowie Haddock/Maio 2014: 199). Einstellungen helfen zunächst dabei, die Umwelt zu strukturieren. Indem sie sich auch auf soziale Kategorien beziehen, können sie sowohl der Konstitution von Zugehörigkeit als auch der sozialen Abgrenzung dienlich sein. Darüber hinaus können Einstellungen in das Selbstkonzept eines Individuums aufgenommen werden oder es begründen, womit sie gleichermaßen auf personale Identitätskonstruktionen Bezug nehmen (zu den Funktionen von Einstellungen in Anlehnung an Katz 1960 vgl. Haddock/Maio 2014: 208f. sowie Bergler/Six 1972: 1403-1405).

Die unterschiedlichen Bestimmungen des Einstellungsbegriffs rücken teilweise verschiedene Komponenten in den Vordergrund. So versteht Allport (1935: 810) unter Einstellung einen auf Erfahrung basierenden, mentalen oder neuralen Bereitschaftszustand, während Eagly/Chaiken den Bewertungsaspekt der Einstellung betonen und sie als ,a psychological tendency that is expressed by evaluating a particular entity with some degree of favor or disfavor" (Eagly/Chaiken 1993: 1) definieren. Es hat sich - nicht zuletzt auch in der linguistischen Einstellungsforschung - ein Mehrkomponentenmodell etabliert, das nach Rosenberg/Hovland (1960) drei grundlegende Komponenten umfasst: (a) eine kognitive Komponente, die Wissensbestände und

\footnotetext{
${ }^{11}$ Silverstein (2003) differenziert in diesem Zusammenhang zwischen verschiedenen Stufen der Indexikalität sprachlicher Merkmale, die sich darin unterscheiden, inwieweit Sprachbenutzer*innen sich der sozialen Bedeutung der Formen, also ihrer Verbindung zu sozialen Gruppen, bewusst sind oder sie bewusst einsetzen. Varianten, die von Sprachbenutzer*innen als zu sozialen Gruppen zugehörig wahrgenommen werden (,n+1 -th-order indexical“" nach Silverstein (2003); vgl. hierzu auch Johnstone/Andrus/Danielson (2006: 82-83)), fallen nach Agha (2005: 38) unter das Konzept Enregisterment, ,,processes whereby distinct forms of speech come to be socially recognized (or enregistered) as indexical of speaker attributes by a population of language users.“ Vgl. zur Begriffsbestimmung und -übersetzung Spitzmüller (2013: 267f.) und Jürgens (2015a: 8-12).

${ }^{12}$ Zur Verknüpfung lokaler Sprachvarianten mit sozialen Identitätskonzepten am Beispiel des Pittsburghese vgl. Johnstone/Andrus/Danielson (2006); am Beispiel des Niederdeutschen vgl. Neumann/Schröder (2017a).
}

ISSN 1615-3014 
Überzeugungen beinhaltet, (b) eine affektive Komponente, in der sich Gefühle gegenüber dem Einstellungsobjekt spiegeln sowie (c) eine konative Komponente, welche sich in spezifischen Verhaltensweisen zeigt (vgl. zusammenfassend Lasagabaster 2004: 400 sowie Haddock/Maio 2014: 199-206 im Anschluss an Zanna/Rempel 1988). ${ }^{13}$

Das Konzept wird in der Linguistik auf sprachliche Erscheinungsformen angewendet, die als Einstellungsobjekte fungieren (vgl. Neuland 1993: 729). Einstellungen gegenüber Sprache implizieren Meinungen und Wertungen, welche die sprachliche Wirklichkeit erschließen und ordnen (Arendt 2010: 7). Bei Spracheinstellungsäußerungen handelt es sich damit um subjektive Daten, welche von objektiven Daten, also sprachlichen Äußerungen, die sich nicht auf Sprache beziehen, abzugrenzen sind (vgl. Neuland 1993: 723f.). Die kognitive Komponente steht mit sprachlichen Wissensbeständen, Vorstellungen oder Konzeptualisierungen in Verbindung. Gefühle sowie emotive Zuschreibungen, wie z. B. Sympathie gegenüber einer Varietät, bilden die affektive Komponente der Spracheinstellung. Dagegen rekurriert ihre konative Komponente auf das sprachliche Verhalten, indem bestimmte Sprachformen bspw. gemieden oder genutzt werden (vgl. Neuland 1993: 728).

Eine Analyse von (Sprach-)Einstellungen ermöglicht eine Rekonstruktion von Identitätskonzepten, da Zuschreibungen und Bewertungen mit Selbstbildern und sozialen Zugehörigkeiten zusammenhängen (vgl. Schröder/Jürgens 2017: 14). ${ }^{14}$ Dabei können auch Stereotype von Bedeutung sein, die als gesellschaftlich geteilte Einstellungsformen aufgefasst werden können (zum Stereotypenbegriff sowie zur Einbettung in das Konzept der Einstellung vgl. Schröder/Jürgens 2017: 21-25).

\subsection{Positionierung}

Im Rahmen sozialer Interaktionen können Personen spezifische Aspekte ihrer Identität hervorbringen, indem sie ,,ihre jeweiligen Positionen festlegen, beanspruchen, zuweisen und aushandeln“ (Lucius-Hoene/Deppermann 2002: 196). So bezeichnet der Begriff Positionierung ,zunächst ganz allgemein die diskursiven Praktiken, mit denen Menschen sich selbst und andere in sprachlichen Interaktionen aufeinander bezogen als Personen her- und darstellen“ (LuciusHoene/Deppermann 2004: 168; vgl. auch grundlegend Davies/Harré 1990). Positionierungen können sich auf bestimmte Attribute, Eigenschaften oder Motive beziehen, soziale Rollen zuoder abweisen sowie auf moralische Ordnungen rekurrieren (vgl. Lucius-Hoene/Deppermann 2002: 199). Sie können implizit wie explizit ausgedrückt werden und sind nicht an bestimmte sprachliche Handlungen gebunden (vgl. Lucius-Hoene/Deppermann 2002: 196-199).

Durch Positionierungen verdeutlichen Sprechende einerseits, wie sie von anderen wahrgenommen werden wollen (Selbstpositionierung). Andererseits zeigen sie, wie sie ihre Gesprächs-

\footnotetext{
${ }^{13}$ Bezogen auf die Fans eines Fußballvereins wie dem FC St. Pauli oder dem HSV kann sich eine positive Einstellung auf unterschiedliche Weise abzeichnen, bspw. in Form der Überzeugung, einer Stadt und ihrem Verein anzugehören (kognitive Ebene), in positiven und negativen Emotionen, die durch sportliche Erfolge oder Misserfolge ausgelöst werden (affektive Ebene) oder im (lautstarken) Unterstützen der Mannschaft während eines Spiels (konative Ebene) (vgl. ähnlich Lenhard 2002: 85-113; eine quantitative Sicht auf die Einstellungskomponenten mit dem Bezugsobjekt Fußball liefert Herrmann 1977: 50-75).

${ }^{14}$ Tophinke/Ziegler (2006: 206) plädieren für ein kontextsensitives Modell zur Untersuchung von Spracheinstellungen, welches soziokulturelle, situative sowie interaktionale Rahmenbedingungen mitberücksichtigt.
}

ISSN 1615-3014 
partner*innen sehen (Fremdpositionierung; vgl. Lucius-Hoene/Deppermann 2002: 62). Ein Beispiel aus dem zu analysierenden Gruppeninterview soll dies veranschaulichen: Als GP01 (weiblich, *1972) in der Diskussion über vereinsspezifische Musik und Fangesänge eine prüfende Frage an die Interviewerin (*1989) richtet („Haste denn schon ma Abschlach! ${ }^{15}$ gehört?"“), nimmt sie beidseitige Positionierungen vor, indem sie die bestehenden Rollen, die der Interviewerin als Fragestellerin sowie ihre eigene als Antwortende, aufhebt.

Eine besondere Bedeutung kommt der Praktik in autobiographischen Erzählungen zu (vgl. Bamberg 1997: 336); damit ist sie mit dem Konzept der narrativen Identität verbunden, welche „eine lokale und pragmatisch situierte Identität [bezeichnet; L. N.], die durch eine autobiographische Erzählung hergestellt und in ihr dargestellt wird“ (Lucius-Hoene/Deppermann 2002: 55). In der Narration kommen verschiedene Positionierungsebenen zum Vorschein: So können Positionierungen sowohl das erzählte Ich sowie die in der Erzählung angeführten Personen als auch den*die Sprecher*in und die anwesenden Personen im aktuellen Erzählkontext betreffen (vgl. Lucius-Hoene/Deppermann 2002: 63; vgl. ferner Bamberg 1997: 337). ${ }^{16}$

\section{$4 \quad$ Untersuchungsdesign}

Im Rahmen der Studie werden je Verein vier Fangruppen untersucht, die jeweils eine Gruppengröße von drei bis vier Personen aufweisen. Sie wurden u. a. durch Aushänge und Kleinanzeigen, durch das Kontaktieren von Fanclubs sowie durch das Schneeballprinzip akquiriert. Die Gruppen bilden soziale Netzwerke, indem sie sich regelmäßig gemeinsam die Spiele ihres Vereins anschauen, sich über Fußball austauschen und ihre Freizeit anderweitig miteinander verbringen. Ihren Lebensmittelpunkt stellt die Stadt Hamburg dar, in welcher die Gewährspersonen auch (sprachlich) überwiegend sozialisiert sind. ${ }^{17}$ Aufgrund der z. T. großen Altersunterschiede in Fannetzwerken werden Personen im Alter zwischen 18 und 60 Jahren befragt. Jede Fangruppe durchläuft zwei oder drei verschiedene Erhebungssituationen.

Zunächst werden mithilfe eines Fragebogens Informationen erhoben, die einen ersten Einblick liefern, welche Bedeutung die Fans dem Fußball bzw. ihrem Verein im Alltag beimessen. Diese Rahmendaten machen sichtbar, welche speziellen Eigenschaften oder Verhaltensweisen mit der sozialen Zugehörigkeit jeweils verbunden werden. Es werden insgesamt fünf Bereiche abgedeckt: Im Hinblick auf die Fanbiographie wird z. B. gefragt, seit wann die Gewährspersonen bereits Fans ihres Vereins sind und wie bzw. wodurch sie es geworden sind. Unter den Aspekten Konsum und Informationsbeschaffung fallen Fragen, welche die Medien und die Nutzungshäufigkeit betreffen, über die sich die Fans informieren oder Fußballspiele verfolgen. Die Fragen zum Fannetzwerk befassen sich mit Mitgliedschaften und Aktivitäten in Fanclubs, Tippgemeinschaften oder Facebook- und WhatsApp-Gruppen. In Bezug auf außersprachliche Komponenten der Identitätsmarkierung wird u. a. gefragt, ob und woran in der eigenen Wohnung, dem Arbeitsplatz, dem Auto oder einem selbst (durch Bekleidung, Accessoires oder Tattoos) die

\footnotetext{
${ }^{15}$ Abschlach! ist eine sechsköpfige Punkrockband aus Hamburg, die sich Mitte der 1990er Jahre gegründet hat und deren Musik im Besonderen auf den HSV und die Stadt Hamburg Bezug nimmt.

${ }^{16}$ Obwohl im Dissertationsprojekt keine narrativen Interviews durchgeführt werden, können (mehr oder weniger wohlgeformte) Erzählungen von den Gewährspersonen dazu genutzt werden, personale oder soziale Identität narrativ zu konstruieren.

${ }^{17}$ Unterschiede in den sprachlichen Sozialisierungen werden berücksichtigt.
}

ISSN 1615-3014 
Vereinszugehörigkeit erkannt werden kann. Abschließend wird der Fragebogen genutzt, um Metadaten wie das Alter, den Geburtsort und die Erstsprache(n) zu erfassen.

In der zweiten Erhebungssituation schauen sich die Fangruppen ein live-übertragenes Fußballspiel ihres Vereins an. Die Kommentare während des Fußballspiels und in der Halbzeitpause werden mit einem Aufnahmegerät aufgezeichnet. Zweck des Settings ist es, spontane, natürliche Sprachdaten der Gewährspersonen zu generieren. Pro Verein wird die Situation Fußballspiel schauen bei zwei der vier Gruppen erhoben, nämlich bei solchen, die (Auswärts-)Spiele üblicherweise am Fernsehgerät zu Hause oder in einer Sportbar verfolgen. Die objektsprachlichen Daten liefern einen Einblick in den Sprachgebrauch (in einer emotional aufgeladenen Situation) und darüber, welche (regional-)sprachlichen Phänomene verwendet und ggf. mit auBersprachlicher Bedeutung belegt werden. Gleichzeitig können diskursive Strategien der Abgrenzung ermittelt werden.

Das Kernstück der Studie bildet ein leitfadengestütztes Gruppeninterview, das mit allen acht Fangruppen geführt wird und nach Möglichkeit in einem vertrauten Setting (Räumlichkeiten des Fanclubs, Stammlokal etc.) stattfindet. Im Interview werden zunächst Rahmendaten zur jeweiligen Fangruppe erhoben, z. B. woher und wie lange sich die Personen kennen. Daran anschließend werden ihre Rituale thematisiert, um einen Einblick in gruppenspezifische Verhaltensweisen zu erhalten. Die Fangruppen, die sich im Rahmen der Studienteilnahme kein Fußballspiel anschauen, werden außerdem nach besonders emotionalen, fußballbezogenen Erlebnissen gefragt, um eine narrative Sprachprobe zu erhalten, die im Hinblick auf Identitätskonstruktionen untersucht werden kann. Unter dem Aspekt Fanidentität soll festgestellt werden, woran ein (echter) Fan erkennbar ist und inwieweit die Gewährspersonen sich selbst und andere als solche wahrnehmen. Dabei wird auch auf außersprachliche Komponenten Bezug genommen, indem durch Fotos konkrete Einstellungen gegenüber spezifischen Gruppen- und Vereinszugehörigkeiten ${ }^{18}$ elizitiert werden, die in der Öffentlichkeit auf Autos oder in Form von Graffitis oder Tattoos zutage treten. Darüber hinaus wird die Lokalrivalität diskutiert, um Praktiken der sozialen Abgrenzung zu erschließen. Der Interviewabschnitt, der sich mit sprachlichen Identitätskonstruktionen befasst, wird damit eingeleitet, dass den Gruppen jeweils zehn Screenshots von Facebook-Statusmeldungen ihres Vereins ${ }^{19}$ gezeigt werden, die vom schriftlichen Standard abweichen. Die Fangruppen werden im ersten Schritt nach (sprachlichen) Auffälligkeiten in den Statusmeldungen gefragt. Damit sollen subjektive Sprachdaten - insbesondere metasprachliche Wissensbestände - erhoben werden, wobei die Aufmerksamkeit der Gewährspersonen vom eingangs schriftlichen Impuls in Richtung mündliche Ausdrucksweisen gelenkt wird. Zudem soll das Identifikationspotenzial der Sprachwahl durch gezielte Fragen überprüft werden (,Fühlt ihr euch dadurch angesprochen?“, „Passt das auch zu euch?“). Im Anschluss findet eine Bewertung der Sprachwahl statt, wobei das semantische Differential aus

\footnotetext{
${ }^{18}$ Neben einigen Fanclubs, die ihr Logo z. B. auf Kleidungsstücken abbilden, ist auf öffentlich sichtbare Symbole von Ultra-Gruppen wie Aufkleber oder Tags zu verweisen. Zur Genese und Struktur der Ultraszene vgl. zusammenfassend Kotthaus (2017).

${ }^{19}$ Für beide Vereine liegen jeweils sieben Statusmeldungen vor, die dieselben regionalsprachlichen Merkmale enthalten. Die übrigen drei Statusmeldungen weichen auf andere Art vom kodifizierten schriftlichen Standard ab. Die Erhebungsmethode sowie die Phänomene aus den Facebook-Statusmeldungen des HSV werden in Kap. 5 weiter ausgeführt.
} 
dem NiH-Fragebogen ${ }^{20}$ verwendet wird. Dadurch können die Sprachbewertungen unmittelbar mit denen einer größeren Kontrollgruppe verglichen werden. Zum Abschluss werden Ortseinstellungen zur Stadt Hamburg erhoben, um regionale Identitätskonzepte zu fokussieren. Dabei wird nach der Beliebtheit des Wohnortes sowie nach Orten, wo sich die Gewährspersonen besonders zu Hause fühlen, gefragt; es werden Auto- und Heterostereotype, die im Vergleich zu Personen aus anderen Großstädten sichtbar werden, sowie das Interesse am gesellschaftlichen und politischen Geschehen Hamburgs eruiert.

\section{Beispielanalyse}

Die folgende Analyse eines Gruppeninterviews mit HSV-Fans soll einen exemplarischen Einblick geben, auf welche Weise der hamburgische Substandard in (sprachliche) Identitätskonstruktionen eingebettet wird. ${ }^{21}$ Den Interviewpartner*innen wurde ein Ausdruck mit insgesamt zehn Facebook-Statusmeldungen des HSV vorgelegt, der regionale bzw. konzeptionell mündliche Sprachmerkmale enthält. ${ }^{22}$

Das damit initiierte metasprachliche Gespräch ${ }^{23}$ nimmt drei Aspekte in den Blick: Als Erstes werden Konzeptualisierungen des hamburgischen Substandards untersucht, die sich aus der Diskussion der Facebook-Statusmeldungen ergeben und die regionale Zugehörigkeit der Gesprächspartner*innen zum Ausdruck bringen. Zusätzlich wird der situationsspezifische mündliche Sprachgebrauch beleuchtet. Als Zweites werden Positionierungen in den Blick genommen, die eine Verbindung zwischen dem Hamburgischen (sowie den damit verbundenen Eigenschaften) und den Identitätskonzepten der Interviewpartner*innen nachweisen, die

\footnotetext{
${ }^{20}$ Im Fragebogen des an der Universität Hamburg durchgeführten DFG-Projekts „Einstellungen gegenüber regionalen Sprachformen in der Großstadt: Niederdeutsch in Hamburg (NiH)“ (Projektleitung: Prof. Dr. Ingrid Schröder; Projektlaufzeit: 01.06.2014-31.03.2018) wurden die Bewertungen zum hamburgischen Substandard sowie zum Niederdeutschen anhand eines semantischen Differentials elizitiert, vgl. Kap. 5. In der Studie wurden die Spracheinstellungen von insgesamt 689 Personen erhoben, deren Lebensmittelpunkt die Stadt Hamburg bildet (vgl. hierzu Schröder/Jürgens 2017: 30-32 und Neumann/Schröder 2017: 228f.).

${ }^{21}$ Die Gesprächsausschnitte stammen aus einem Interview mit HSV-Fans, das im April 2018 geführt wurde. Das Ehepaar GP01 (weiblich, *1972 in Hamburg) und GP02 (männlich, *1970 in Hamburg) sind Gründungsmitglieder eines offiziellen HSV-Fanclubs, durch welchen sie GP03 (männlich, *1983 in Dortmund) kennengelernt haben. Beim Interview anwesend war darüber hinaus die Ehefrau der GP03 (*1989 in Hamburg), welche zwar nicht fußballinteressiert, aber auch mit dem Ehepaar GP01 und GP02 befreundet ist.

Das der Analyse zugrundeliegende Interview wurde mithilfe des Partitur-Editors EXMARaLDA (http://exmaralda.org/de/) nach den Konventionen der halbinterpretativen Arbeitstranskription (HIAT) transkribiert (vgl. grundlegend Ehlich/Rehbein 1976).

${ }^{22}$ Bei den Statusmeldungen handelt es sich um Beiträge des HSV, die in den Jahren 2017 und 2018 auf der offiziellen Facebookseite des Vereins (https://www.facebook.com/HSV/) veröffentlicht wurden und folgende Sprachvarianten enthalten: „Moin Moin“ (08.04.2017), „Bidde“ (19.05.2017), „Schietwetter“ (08.09.2017), „Lütten“ (14.12.2017), „Kinners“ (20.12.2017), „Denn man tau!“ (02.01.2018), „N“ Eis“ (02.01.2018), „Jungs \& Deerns“ (11.02.2018), „Endlich geiht dat los!“ (07.03.2018). Mitgepostete Bilder wurden den Fans lediglich in einem Fall gezeigt, in welchem die Sprachmerkmale im Bildtext integriert sind („NICH“ LANG SCHNACKEN, VERSANDKOSTENFREI BESTELLEN!““(04.09.2017)).

${ }^{23}$ Die methodische Herangehensweise schließt damit zwar an wahrnehmungsdialektologische Methoden an (zu verschiedenen methodischen Vorgehensweisen in der Wahrnehmungsdialektologie vgl. Preston 2010), der verwendete Stimulus ist im Gegensatz zu anderen Studien, besonders solchen aus der Salienzforschung (u. a. Hettler 2018; Hundt/Palliwoda/Schröder 2017), jedoch nicht akustisch wahrnehmbar, sondern eindeutig als schriftliche Abweichung vom (hochdeutschen) Standard zu identifizieren.
} 
diastratisch begründet werden. Als Drittes werden die Bewertungen des hamburgischen Substandards durch die befreundete Gruppe den Ergebnissen des semantischen Differentials des $\mathrm{NiH}-P$ rojekts gegenübergestellt, wodurch die Bewertungen in einem erweiterten Kontext betrachtet und zugleich weitere sprachbezogene Identitätskonstruktionen aufgezeigt werden können. ${ }^{24}$

$\mathrm{Zu}$ diesen Zwecken werden mehrere Analysemethoden miteinander kombiniert. Mithilfe von inhaltsanalytischen Verfahren (vgl. Mayring 2015) können insbesondere regionalsprachliche Wissensbestände und Spracheinstellungen ermittelt werden. ${ }^{25}$ Zusätzliche Analysen der Positionierungsverfahren (vgl. Kap. 3.3) rekonstruieren zudem die Selbst- und Fremdbilder der Gewährspersonen, die sich sowohl in referierten Sprechsituationen als auch in der Interviewsituation unter den anwesenden Teilnehmer*innen abzeichnen. Gleichzeitig wird die sprachliche Realisierung in den Blick genommen. So werden gesprächsanalytische Verfahren (vgl. König 2014; Jürgens/Schröder 2016: 355-356) dazu genutzt, die sprachlichen Merkmale aufzudecken, die die Konzeptualisierungen und Identitätskonstruktionen implizit wie explizit hervorbringen.

\subsection{Konzeptualisierungen des Hamburgischen}

Als Antwort auf die Frage, was den Gewährspersonen an den Statusmeldungen auffällt, werden von diesen zunächst die Inhalte der Statusmeldungen in den Blick genommen und einzelne angesprochene Themen bewertet (GP03: „die Virtuelle Bundesliga [...] da bin ich ja sowieso total für zu haben für son Scheiß.“; GP03: „,Vi/ viermal schlafen, dann is Weihnachten.“ Wennde nix zu sagen hast, dann sachs ha/ sachst halt sowas."). Erst als die Interviewerin nachfragt, ob und warum die Statusmeldungen nicht vom FC Bayern München sein könnten, und damit den Fokus auf regionale Sprachmerkmale lenkt, stellt GP03 bezüglich der sprachlichen Form fest:

[1]

GP03: Ja gut, da is halt viel Hamburger Schnack mit drin, ne. • Da wird dann halt nich stehen

I:

Hmhm.

[2]

GP01:

Ja.

„Und Buams.“

GP03: „Jungs und Deerns aufgepasst“, sondern •• „Madels und...“ Keine Ahnung. „Buams.“

I:

$\mathrm{Hm}$.

[3]

\footnotetext{
${ }^{24}$ Die Sprachbewertungen wurden mithilfe der Frage 15 ,Welche der folgenden Eigenschaften verbinden Sie persönlich am ehesten mit einer typisch hamburgischen Umgangssprache?“ erhoben. Die neun Eigenschaftspaare, zu welchen die Studienteilnehmer*innen ihre Einschätzung abgeben sollten, lauten: „humorvoll - ernst“, „,vornehm grob“, „gemütlich - ungemütlich“, „vertraut - distanziert“, „,freundlich - unfreundlich“, ,modern - altmodisch“, „sympathisch - unsympathisch“, „,cool - uncool“ und „geradeheraus -umständlich“. Optiert werden konnte auf einer fünfstufigen Skala, sodass auch eine neutrale Antwort möglich war.

${ }^{25}$ In diesem Zusammenhang sind bisherige Studien zum Hamburgischen (Hettler 2016; Hettler 2018; Jürgens 2015b) und zum Niederdeutschen in Hamburg (Schröder 2010; Jürgens 2015a; Neumann/Schröder 2017b) miteinzubeziehen.
} 
GP01: Genau.

GP03: Oder nich „Moin Moin“, sondern „Grüezi mitenand“.

Die regionalen Phänomene werden von GP03 als „Hamburger Schnack“ eingeordnet und einzelne Merkmale (,Jungs und Deerns“, „Moin Moin“) der bairischen („Madels und...“ Keine Ahnung. „Buams.“) bzw. schweizerdeutschen Variante („Grüezi mitenand“) gegenübergestellt. Als weiterer Dialektraum und abzugrenzende Sprecher*innengruppe werden darüber hinaus „die Sachsen“ (GP03) genannt, bei welchen der Interviewpartner ebenso (schrift-)sprachliche Abweichungen vermutet. Anders als bei den sprachlichen Erscheinungsformen sind sich die Gewährspersonen in Bezug auf die Inhalte der Statusmeldungen dagegen einig, dass zwischen den beiden Vereinen keine Unterschiede zu erwarten seien (GP01: „Aber vom/ • a aber vom Inhalt könnten sie • ein und dasselbe sein.“).

Die mäßige Auffälligkeit der Sprachwahl erklären sich die Gewährspersonen vor allem durch den alltäglichen Gebrauch (GP03: „Ja, du schnackst halt so den ganzen Tach, ne.“, GP01: „Weil das isso als Hamburger, $\cdots$ das is so der Sprachgebrauch.“), die auch in Abgrenzung zu anderen Dialekträumen veranschaulicht wird:

GP01:

$\mathrm{Hm}$.

GP03: Ähm also was heißt aufgefallen, man liest es halt hier irgendwie so mit, ne.

I:

$\mathrm{Hm}$.

[2]

GP02:

-. Ich mein, wenn da/ wenn da...

GP03: Wenn du, ich sag jetz mal, als/

(Schumpen-) ${ }^{26}$ Scheißer hier hoch

[3]

GP02:

Oder wenn

GP03: kommst, und liest so öh/ da wirst auch erst mal sagen: „Hä, wie ,Kinners“?“

[4]

GP02: das jetz/ wenn das eher mit diesem Bairischem Dia/ Dialekt, denn/ denn

GP03:

- Dann würd's dir auffallen.

[5]

GP02: würde man/ denn würde man eher sagen: „Wat schreiben die fürn Scheiß.“

GP03:

Aber das is halt $\mathrm{Zu}-$

hausesprache.

Die regionalsprachlichen Merkmale in den Statusmeldungen des HSV werden laut GP03 in der eigenen Region (,hier") mitgelesen, ohne dass ihnen weitere Beachtung zuteil wird („Ähm also was heißt aufgefallen“). Dagegen erwartet der Interviewpartner, dass die Varianten von einem sogenannten ,(Schumpen-)Scheißer“, welcher hier als Stereotyp für eine

\footnotetext{
${ }^{26}$ Klammerungen im Transkript stehen für vermutete Äußerungen, die schwer verständlich sind.
} 
Person aus dem süddeutschen (und ländlich geprägten) Dialektraum angeführt wird, bemerkt würden. Die Auffälligkeit des regionalen Sprachgebrauchs hängt für GP03 folglich von der Herkunftsregion der rezipierenden Person ab. Die Ausführungen von GP02 bestätigen den Zusammenhang. Die Vorstellung, bairische Merkmale in den Statusmeldungen des HSV zu beobachten, führt zu dem Schluss: „denn würde man eher sagen: ,Wat schreiben die fürn Scheiß.“" GP02 wertet den Gebrauch eines fremden Dialekts nicht nur mit dem Ausdruck „Scheiß“ explizit ab, sondern stellt sich durch die Verwendung des regiolektalen „Wat“ gleichzeitig der eigenen Region als zugehörig dar. Diese Zugehörigkeit wird auch von GP03 ausgedrückt, nämlich mit der Wahl des Begriffs „Zuhausesprache“.

Die verwendete Sprache, die GP03 eingangs als „Hamburger Schnack“ bezeichnete, wird von GP01 als „Son bisschen/ bisschen ins Plattdüütsche so. Ja, Hamburgisch.“ beschrieben. GP03 bestätigt dies und macht zusätzlich auf die konzeptionelle Nähe zum Mündlichen aufmerksam: „Ja, und das is halt auch (dieses) ,N' Eis geht immer', Lautsprache, ne.“ Im weiteren Gesprächsverlauf werden von den Gewährspersonen zudem die Bezeichnungen „Platt““ (GP03), „Hamburger Slang“ (GP01) und „Hafenslang“ (GP03) verwendet (vgl. zu weiteren Benennungen Jürgens 2015b: 190f. und Hettler 2016: 189f.).

Als im Rahmen des Gesprächs die in Hamburg wahrgenommene Sprachlage in den Fokus rückt, wird das Verhältnis von Hochdeutsch und Plattdeutsch im mündlichen Sprachgebrauch skizziert:

[1]

GP01:

Ja.

GP03:

Ja. Platt $\cdots$ und graderaus, also du

I: $\quad[\ldots]$ Was fällt euch da noch so ein, wie man spricht? Ähm...

[2]

GP03: sachst halt so...

I:

- - Aber wer redet denn/ also... • Aber hört ihr Platt auf der Straße?

[3]

GP01: Ja.

GP03: Ja. Also irgenw/ au/ auffer Stra/ beziehungsweise auf der Arbeit, Baustelle. Bei mir [4]

GP01:

Ja, bei mir im Hafen. Ja.

GP03: isses jetz zum Beispiel so. Da wird ganz viel Platt geschnackt.

Das

[5]

GP02: Das is ja immer son/ das is ja son Stückwerk • • von Hochdeutsch und Platt. Das

GP03: is so...

$\mathrm{Ja}$, genau! Genau, das is

[6]

GP02: is ja/ das sind ja immer so ein/ einzelne Worte (oder) Silben, die darin/

GP03: son Misch/ so, wie sie's hier schreiben quasi. „Kinners, nur noch viermal schlafen, dann 
[7]

GP02: die denn halt auf Platt rüberkommen.

- Also wenn/ wenn man aufm Dorf is un/

GP03: is Weihnachten.“ Ja.

Ja.

I:

Hmhm.

[8]

GP02: un/ und die äh • sprechen nur Platt, $\bullet$ das is denn schon wieder ganz was andres.

Als weitere Assoziationen mit der Sprachlage in Hamburg nennt GP03 die Begriffe „Platt • • • und graderaus", die erklärende Ausführung (,,also du sachst halt so...") wird vom Sprecher abgebrochen. Die Interviewerin fragt daraufhin nach, ob die Gruppe „Platt“ auch in der Öffentlichkeit (,auf der Straße“) bemerkt. Sowohl GP03 als auch GP01 reagieren zustimmend (,Ja.“). Erst im nächsten Schritt wird der Kontext von GP03 auf den Arbeitsbereich (,,beziehungsweise auf der Arbeit, Baustelle“) eingeschränkt, anschließend durch die Präpositionalphrase „bei mir“ subjektiviert sowie als Beispiel deklariert. Die Beobachtungen im eigenen Arbeitsumfeld werden von GP03 zum Abschluss seines Redebeitrags bekräftigt („Da wird ganz viel Platt geschnackt.“). Dem Wahrheitsgehalt des Gesagten wird durch die Wortwahl ,geschnackt" Nachdruck verliehen, indem der niederdeutschgeprägte Sprachgebrauch bei der Arbeit abgebildet wird. Die Wahrnehmung im beruflichen Kontext wird daraufhin von GP01 übernommen (,Ja, bei mir im Hafen."). Eine Einordnung der bis zu diesem Zeitpunkt noch nicht weiter eingegrenzten Sprachlage liefert GP02 (,son Stückwerk • • von Hochdeutsch und Platt") und macht dabei auf unterschiedliche grammatische Ebenen (,ein/ einzelne Worte (oder) Silben“) aufmerksam, welche als Niederdeutsch rezipiert werden. GP03, der GP02 ausdrücklich zustimmt („Ja, genau!“), beginnt simultan mit einem Erklärungsansatz („son Misch/“), der schließlich mit dem Verweis auf eine der Statusmeldungen als Exempel für die Sprachlage endet. Die Ausführungen beider Gesprächspartner werden von der Interviewerin („Hmhm.“) sowie von GP03 selbst („Ja.“) bestätigt. Nach kurzer Pause erläutert GP02 weiter: „Also wenn/ wenn man aufm Dorf is un/ un/ und die äh • sprechen nur Platt, • • das is denn schon wieder ganz was andres.“ Diese Äußerung impliziert das verbreitete Stereotyp, dass Niederdeutsch auf dem Land gesprochen werde (vgl. Jürgens/Schröder 2016, die verschiedene Wissensformen unterscheiden, sowie Arendt 2010: 156). Der stereotype Wissensbestand des Interviewpartners dient in dem Redebeitrag dazu, die Sprachlage in der Großstadt Hamburg durch Kontrastierung zu veranschaulichen und abzugrenzen; sprachlich setzt er dies mithilfe eines Konditionalgefüges um.

Die Verwendung am Arbeitsplatz sowie der Vergleich zum Land werden von den Gewährspersonen im Zuge der Thematisierung eines situationsspezifischen Sprachgebrauchs erneut aufgegriffen:

[1]

I: Redet ihr immer so oder findet ihr, dass man • in manchen Situationen eher so reden [2]

GP02: ((schnalzt)) Also auffe Arbeit $\cdots \cdot$ sprech ich eigentlich eher nich so.

I: kann als in anderen?

[3] 
GP01: • Ich ja.

GP02: $\quad$ - Ja, gut. Im Hafen kann man so sprechen und...

GP03: Ich auch. Auffe Baustelle geht

[4]

GP03: das auch. $\quad[\ldots]$ O/ oder ich sach mal, ich hab 'n Kunden irgendwo aufm Dorf und

Ehefrau GP03: ((lacht))

[5]

GP02:

Da gibt's 'n Unterschied. Ja, genau. Ja.

GP03: ich hab 'n Kunden in Eppendorf. Da mussu schon gucken, wie du redest. • Beim

I: $\mathrm{Hm}$.

$\mathrm{Hm}$.

[6]

GP03: Fußball • • de/ d/ d/ da/ da läuft/ d/ d/ da sprichst du halt, wie's grade vonne Zunge rollt.

[7]

GP01: Ja, beim Fußball is halt jeder privat.

GP02: $\quad$ (So isses.) $\quad \mathrm{Hm} . \quad$ ((2s)) Da

GP03: Aber w... Genau(so) sieht's aus, das halt privat, ja.

I: $\quad \mathrm{Hm}$.

[8]

GP02: brauch man sich auch nich so verbiegen. $\quad$ ‥ Überhaupt nich.

GP03:

Nö.

Während GP02 im Beruf das Register wechselt, bestätigen GP01 und GP03 den regionalen Sprachgebrauch für ihr Arbeitsumfeld. GP02 lenkt den Fokus daraufhin auf den Arbeitsort seiner Ehefrau: „• Ja, gut. Im Hafen kann man so sprechen und...“ Inwieweit der Sprecher an dieser Stelle eigenes (stereotypes) Wissen aktiviert oder sich auf eine vorangegangene Aussage von GP01 bezieht, in welcher der Substandard im Hafen verortet wird, kann nicht entschieden werden. Der Turn wird von GP03 übernommen, welcher die abgebrochene Äußerung von GP02 zunächst beendet („Auffe Baustelle geht das auch.“). GP03, der einen handwerklichen Beruf mit flexiblen Arbeitsorten ausübt, schränkt den Geltungsbereich anschließend ein, indem er seine Kundschaft vom Land der Kundschaft aus dem Hamburger Stadtteil Eppendorf gegenüberstellt. ${ }^{27}$ Seine Beobachtung, dass er in Eppendorf auf seinen Sprachgebrauch Acht gebe, formuliert GP03 generalisierend in der zweiten Person Singular („Da mussu schon gucken, wie du redest"). Das Modalverb (,mussu“) deutet außerdem an, dass die Gründe für einen notwendigen Registerwechsel von seiner Umgebung bestimmt werden, was GP02 simultan bestätigt. Im Anschluss wird der Fußballkontext als informelle Situation kontrastiert, in der gesprochen werde, ,wie's grade vonne Zunge rollt.“ Der niedrige Formalitätsgrad wird von GP01 bekräftigt; sie beschreibt die Situation als eine, in der ,jeder privat" sei. GP02 greift den weiteren

\footnotetext{
${ }^{27}$ Hettler (2018: 204) verweist in diesem Zusammenhang auf die Einteilung in feine und unfeine Hamburger Stadtteile. Mit Bezug auf den Stadtteil Eppendorf vgl. Hettler (2016: 183).
} 
Aspekt auf, dass man sich beim Fußball „auch nich so verbiegen“ müsse, wobei das Adverb „auch“ vermuten lässt, dass sich diese Äußerung nicht ausschließlich auf sprachliches Verhalten bezieht.

Bei der geographischen Zuordnung werden das Niederdeutsche und der lokale Substandard gleichzeitig betrachtet. Als Orte und Stadtteile von Hamburg, in welchen verstärkt regionale Sprachformen verortet werden, führen die Gewährspersonen u. a. die als peripher wahrgenommenen Stadtteile Finkenwerder, Ochsenwerder, Moorburg, zusammenfassend ,alles nah/ du kanns ma sagen, alles nah der Elbe“ (GP03), an, wobei GP01 zusätzlich auf unterschiedliche Bevölkerungsteile („Bauern“, Orte mit niedrigerem Migrant*innenanteil) verweist (vgl. zum Niederdeutschen Schröder 2010: 593f.). Im Hinblick auf den Elbvorort Blankenese sind sich die Interviewpartner*innen zunächst uneinig, bis GP03 konstatiert: „Ähm Blankenese is gar nich so unverkehrt. Da wird viel Pl... Wei/ ja, da wird viel Platt geredet. [...] Auch wenn da das Geld wohnt, sach ich mal [...]" (zu einem vergleichbaren Beleg vgl. Hettler 2016: 193f;; ferner Jürgens 2015b: 197). Darüber hinaus bringt GP01 ihr Bedauern zum Ausdruck, dass der regionale Sprachgebrauch im Stadtteil des Ortsrivalen zu verorten sei (,Ja, San/ San Pauli leider, Kiez, ne.“), was mit einer früheren Äußerung der Sprecherin konform geht, als sie bei der Frage nach dem Zugehören der regionalen Sprache zum FC St. Pauli den Begründungen von GP02 („Das sind ja auch irgendwie Hamburger, ne.“) und GP03 („,Ja, wenn dies benutzen, ja, warum nich. Das is ja auch/ es is ja Hamburg. Wie gesacht, es is ja der Stadtteil einer Stadt, ne.“) scherzhaft widerspricht („Nein. [...] ((lacht 1,4s)) Natürlich nich. ((lacht 0,5s))“).

Der lokale Substandard wird von den Gewährspersonen als frequent genutzt wahrgenommen. So wird als Antwort auf die Frage, welche privaten oder prominenten Sprachbenutzer*innen bekannt sind, zunächst die große Anzahl an Sprecher*innen betont (GP03: „Ne Menge.“). Auf konkrete Nachfrage der Interviewerin nennt GP02 „Loddo King Kaal [Lotto King Karl, mit bürgerlichem Namen Gerrit Heesemann, L. N.], zum Beispiel.“, der als Stadionsprecher und Sänger unmittelbar mit dem HSV in Verbindung steht. Seinen bisherigen Ausführungen entsprechend weitet GP03 die ihm bekannten Sprecher*innengruppen auch auf den Fußballkontext aus („Viele ausm Fanclub, viele im Stadion, beim/ viele auffe Baustelle, keine Ahnung. Viele im Freundeskreis.“), um schließlich hervorzuheben: „Ne Zahl könnt ich dir jetz gar nich nennen.“

\subsection{Positionierungen}

Die Frage nach einem situationsspezifischen Sprachgebrauch entwickelt sich zu einer Diskussion über Sprecher*innengruppen, bei der GP02 festhält: „Also es gibt/ es gibt Leute, die/ die sprechen/ sprechen mehr so, $\bullet$ und es gibt Leute, die sprechen weniger so. Das f/ das fällt schon auf." Auf der Suche nach einem Beispiel für Sprecher*innengruppen, die sich in ihrem Gebrauch von regionalen Sprachmerkmalen unterscheiden, übernimmt GP01 den Turn:

[1]

GP01: Doch, du hast es schon bei/ bei/ bei $\bullet$ Leuten, die in/ in/ in äh $\bullet$ ich sach mal eher so $\bullet$ akademischen Berufen $\bullet$ arbeiten, eher etwas $\bullet$ höhergestellt sind $\bullet$ äh ähm die es/ wo man merkt dann, äh die finden es nich so schicklich, so zu reden. 
Ehefrau GP03: Ne/ ihr redet mehr so als ich. •-・Der Hafenarbeiter und der Bauarbeiter, die [3]

GP01:

$$
\text { - Ja. } \quad((1,2 s)) \text { Ja, aber ich sag dir ganz ehrlich, mir }
$$

Ehefrau GP03: reden mehr so als ich.

[4]

GP01: persönlich isses egal, wen ich vodde Nase hab. Ich rede trotzdem so.

GP01, die einer nicht-akademischen Tätigkeit im Hamburger Hafen nachgeht, weist auf einen Zusammenhang zwischen beruflicher Position und Spracheinstellung hin. Die Outgroup - Akademiker*innen bzw. Personen in höhergestellten Berufen - beschreibt die Interviewpartnerin zurückhaltend durch Subjektivierung (,ich sach mal"), Verwendung von Unschärfemarkern (,,eher so“, „,eher etwas“) sowie dem Vor- und Nachschalten kurzer Sprechpausen (,• akademischen Berufen •“, „• höhergestellt sind •"); die unterstellte Spracheinstellung (,die finden es nich so schicklich so zu reden“) wird generalisierend (,man“) formuliert. Die Ehefrau GP03, die als Einzige der Gesprächsteilnehmer*innen in einem akademischen Beruf arbeitet, bestätigt die Äußerung von GP01, indem sie den Sprachgebrauch der übrigen Befragten ihrem eigenen gegenüberstellt („,Ne/ ihr redet mehr so als ich.“). Die Sprecherin stellt sich damit der von GP01 skizzierten Outgroup als zugehörig dar (Selbstpositionierung). Nach einer kurzen Pause reformuliert sie ihren Beleg für einen berufsspezifischen Sprachgebrauch durch die Auflösung der Deixis ,,ihr“ und verwendet stattdessen stereotyp aufgeladene und distanzierende Bezeichnungen („Der Hafenarbeiter und der Bauarbeiter“), wodurch die Fremdpositionen von GP01 und GP03 zusätzlich betont werden. GP01 bestätigt die Ausführungen der Ehefrau vorerst („Ja.“) und wendet sich dann direkt an sie (,aber ich sag dir ganz ehrlich“), um die Fremdpositionierung zu modellieren: Der Substandard wird zum (personalen) Identitätsmerkmal, auch (oder gerade) wenn er bei anderen ein niedrigeres Prestige genießt (,mir persönlich isses egal, wen ich vodde Nase hab. Ich rede trotzdem so."). Sprachlich wird dies durch die standardabweichende Sprachwahl (,,isses“ „vodde Nase hab“) abgebildet und durch die Adjektive ,ganz ehrlich“ und ,persönlich“ sowie das Adverb „trotzdem“ verstärkt.

Im Gruppeninterview wurde darüber hinaus gefragt, ob die Interviewpartner*innen andere Personen sprachlich als Hamburger*innen identifizieren können. Die Gewährspersonen stimmen dem zu, verorten die Sprachmerkmale aber gleichzeitig weiter in Norddeutschland (GP03: „du muss ja auch weiter nördlich sehen [...] die ham diesen • Schnack ja auch irgendwo mit drin“). Als spezifisch für Hamburg konstatiert GP03 später: „Aber du merkst schon, wenn du mit jemandem direkt aus Hamburg redest. Irgendwie/ das isso/ • • de/ dieser Hafenslang, der kommt immer so mit.“ Der „Hafenslang“ wird von GP01 als „•• Direkt. Da/ ohne Blatt vorm Mund.“ beschrieben, was im weiteren Gesprächsverlauf räumlich ausgeweitet wird (GP01: „Aber wenn man mit jemandem redet, dann - - direkt und graderaus. Das is für mich Hamburgisch."). Dabei entsteht erneut eine Diskussion um bildungsspezifische Unterschiede, die Ehefrau GP03 initiiert. Anders als im vorangegangenen Interviewausschnitt, der sich auf den Gebrauch regionalsprachlicher Varianten bezog, grenzt sie sich nun von der sozialen Kategorie Akademiker*innen ab (,da [zu den anwesenden Interviewpartner*innen; L. N.] kann man mal sagen, wenn man irgendwas scheiße findet und bei den andern [den Akademiker*innen; L. N.] gilt man als Zicke.") und resümiert: 
[1]

GP01:

Nö, ich red zu jedem so.

Ehefrau GP03: Da is/ da is schon 'n Unterschied bei so Akademikern.

[2]

GP01:

Nein, aber ich rede auch mit Aka/ Akademikern

Ehefrau GP03: • • Bissu Akademiker? Nein, du bist ja Hafenarbeiter.

[3]

GP01: so äh. Gibt's im Hafen auch, meine Liebe. Ja.

Ehefrau GP03: $\quad$ Ja, aber du! Du! Ja, aber du redest mit denen so.

[4]

GP01:

- Ja. Da hamse 'n Problem. [...]

Ehefrau GP03: Die ja aber nich so mit dir.

[5]

GP02: $\quad$ Mit denen wollen wir auch gar nich reden.

Komm.: $\quad$ ((kollektives Lachen))

GP01 entgegnet auf die Aussage von Ehefrau GP03, die auf Unterschiede bei Akademiker*innen aufmerksam macht, dass sie ,zu jedem so“ rede, wodurch sie sich erneut als geradlinig in ihrem sprachlichen Verhalten darstellt. Da diese Reaktion nicht zur Initialäußerung passt, entgegnet Ehefrau GP03 mit einer Fremdpositionierung ihrer Freundin, die durch die selbstbeantwortete Frage „•• Bissu Akademiker? Nein, du bist ja Hafenarbeiter.“ realisiert wird (zur Funktion von selbstbeantworteten Fragen vgl. Bücker 2015). GP01 reformuliert ihren Standpunkt (,Nein, aber ich rede auch mit Aka/ Akademikern so äh.“) und setzt der Fremdpositionierung durch Ehefrau GP03 ihr Erfahrungswissen entgegen, dass es auch im Hafen Akademiker*innen gebe. Die besitzanzeigende Anrede „meine Liebe“ weist Ehefrau GP03 außerdem zurecht, welche ihre Äußerung sodann paraphrasiert (,Ja, aber du redest mit denen so. Die ja aber nich so mit dir."). GP01 distanziert sich - nicht zuletzt auch auf sprachlicher Ebene - von der Outgroup („Da hamse "n Problem.“). Der Gesprächsabschnitt wird mit einer ironischen Bemerkung von GP02 beendet, welche die Outgroup als unerwünschte Gesprächspartner*innen abstempelt (,Mit denen wollen wir auch gar nich reden.“), sowie dem einvernehmlichen und gemeinsamen Lachen der Gruppe.

\subsection{Sprachbewertungen}

Einige Sprachbewertungen der Gesprächspartner*innen haben sich bereits herauskristallisiert, wie z. B., dass das Hamburgische mit den Eigenschaften ,graderaus“ oder „direkt" verbunden wird (GP01). Zum Abschluss des metasprachlichen Gesprächsabschnitts haben die Gewährspersonen das semantische Differential aus dem NiH-Fragebogen ausgefüllt, um weitere 
Eigenschaften zu erheben, die mit dem hamburgischen Substandard assoziiert werden. ${ }^{28}$ Die Besprechung der Sprachbewertungen macht den Zusammenhang zwischen Eigenschaftszuschreibungen und sprachbezogenen Identitätskonstruktionen sichtbar.

Die Ergebnisse des NiH-Projekts zeigen, dass der lokale Substandard von der Hamburger Bevölkerung überwiegend „,sympathisch“ (87,8 \%), ,geradeheraus“ (85,9\%), ,humorvoll“" $(81,8$ \%), ,gemütlich“ (80,9\%), „vertraut“ (71,4\%), „freundlich“ (69,8\%) und ,grob“ (49,1\%) wahrgenommen wird (vgl. Neumann/Schröder 2017b: 247), ${ }^{29}$ bei den Eigenschaftspaaren „,modern - altmodisch“ und „,cool - uncool“ überwiegen die neutralen Optionen (48,1 bzw. 51,7 \%; unveröffentlichtes Projektmaterial). Jene Befragungsteilnehmer*innen, die glauben, an ihrer Sprache als Hamburger*in erkennbar zu sein, tendieren zu noch positiveren Bewertungstrends, vor allem bei emotiven Eigenschaften (vgl. Neumann/Schröder 2017b: 248f.).

Die Interviewpartner*innen wählen bei den Paaren „humorvoll - ernst“ und „vornehm - grob“ durchweg die neutralen Optionen. ${ }^{30}$ Als Begründung wird der eigene Sprachgebrauch angeführt (GP03: „Aber ich kann doch nich meinen eigenen Dialekt bewerten, ich sprech ihn doch.“, „Weil es is für mich nich witzig. Weil, wenn mich jemand als witzig bezeichnet, wenn ich mit ihm rede, denk ich: Ja, witzig, aber das is meine Sprache.“). Die übrigen Bewertungen lassen ebenso darauf schließen, dass der Substandard mit dem sprachlichen Alltag der Gewährspersonen verbunden wird (GP01: „D/ das normal!“). Analog zu den Ergebnissen des NiH-Projekts tendieren die Sprecher*innen bei den emotiven Eigenschaften ,gemütlich“ (Stufe 1: GP01, GP02, GP03), „freundlich“ (Stufe 1: GP01) und „sympathisch“ (Stufe 1: GP01; Stufe 2: GP03) eher zum positiveren Pol. Erläutert wird dies durch Aussagen wie „Es is einfacher so zu sprechen. $\bullet$ Weil ich muss mich nich verstellen“(GP03) oder ,[...] weil er [ein gedachter Sprecher, L. N.] sich nich verstellt. Weil er ((schmatzt)) dat so raushaut.“ (GP01). Negative Bewertungsmuster sind in der Gruppe nicht zu erkennen. Zwar wählt GP02 bei dem Paar ,modern - altmodisch" die erste Stufe des eher negativen Pols, begründet dies aber mit einer vermutet niedrigeren Sprecher*innenzahl. Neutrale bis positive Antworten finden sich bei den verbleibenden drei Eigenschaftspaaren „,cool - uncool“ (neutral: GP01, GP03; Stufe 1: GP02), ,geradeheraus - umständlich“ (neutral: GP01; Stufe 1: GP02; Stufe 2: GP03) und „vertraut - distanziert“ (neutral: GP02; Stufe 2: GP01, GP03).

\section{$6 \quad$ Fazit}

Die Beispielanalyse eines metasprachlichen Interviews mit HSV-Fans konnte exemplarisch zeigen, auf welche Weise der hamburgische Substandard konzeptualisiert und innerhalb von Positionierungshandlungen und Sprachbewertungen mit den Identitätskonstruktionen der Sprecher*innen verknüpft wird.

\footnotetext{
${ }^{28}$ Beim Vergleich der Ergebnisse ist zu berücksichtigen, dass die Bewertungen der Gewährspersonen im Interview anders als in der Befragung im NiH-Projekt durch die Interaktion vorgeprägt sind und daher im Kontext des Interviews zu betrachten sind (qualitative Daten). Es liegen Ergebnisse für GP01, GP02 und GP03 vor.

${ }^{29}$ Bei der Darstellung wurden die beiden Stufen eines Merkmals addiert.

${ }^{30}$ Der Unterschied zu der Bewertungstendenz der Kontrollgruppe könnte ein Hinweis darauf sein, dass in der NiHStudie vergleichsweise stärker Stereotype reproduziert wurden. Dies kann an dieser Stelle und ohne weitere Analysen jedoch nicht verifiziert werden. (Zum Stereotyp „Plattdeutsch ist humorvoll“ vgl. Jürgens/Schröder 2016: 358-364. Die Ergebnisse von Neumann/Schröder (2017b: 247) weisen eine signifikante Korrelation zwischen der Bewertung des Niederdeutschen und des Hamburgischen nach.)
} 
Die Konzeptualisierungen der thematisierten Sprachlage rekurrieren auf die regionale Zugehörigkeit der Gesprächspartner*innen, welche einerseits explizit ausgedrückt wird (GP03). Andererseits wird emblematisch auf regionale Varianten zurückgegriffen, welche den eigenen Sprachgebrauch sowie die Zugehörigkeit zur Sprecher*innengruppe in der Interviewsituation konstituieren, also mit einer sozialen Bedeutung belegt werden (GP02, GP03). Die Diskussion um eine orts- und situationsspezifische Sprachverwendung deckt notwendige Registerwechsel in bestimmten beruflichen Situationen (GP02) und Stadtteilen (GP03) auf, die dem privaten Sprachgebrauch (GP01, GP03) gegenübergestellt werden. Dabei zeichnen sich Parallelen zu bisherigen Untersuchungen zum Hamburgischen sowie zum Niederdeutschen in Hamburg ab, die auf geteilte (stereotype) Spracheinstellungen hinweisen: Das Land-Stereotyp wird abgerufen (vgl. Jürgens/Schröder 2016), regionales Sprechen wird im und um den Hamburger Hafen verortet (vgl. Schröder 2019 und Hettler 2016) und der Stadtteil Eppendorf wird mit anderen Teilen Hamburgs kontrastiert (vgl. Hettler 2016).

Darüber hinaus werden Positionierungen vorgenommen, die sich auf schichtspezifische Unterschiede (Akademiker*innen versus Nicht-Akademiker*innen) im Sprachverhalten beziehen (GP01, Ehefrau GP03). Das Hamburgische sowie die mit den Sprecher*innen verbundenen Eigenschaften dienen sowohl in der referierten Situation als auch unter den Gesprächspartner*innen im Interview dazu, Selbst- und Fremdpositionen diskursiv zu gestalten (GP01, Ehefrau GP03). Der Outgroup unterstellte Spracheinstellungen werden genutzt, um den eigenen Sprachgebrauch zu begründen und personale Identität zu konstruieren (GP01). Damit deutet sich an, dass dem Hamburgischen ein (verdecktes) Prestige unter Sprecher*innen zukommt (vgl. Schröder 2010 zur sozialsymbolischen Funktion des Niederdeutschen in Hamburg; zum Hamburgischen vgl. Schröder 2015: 49-51 und Hettler 2018: 207f.).

Der Zusammenhang zwischen den Identitätskonzepten der Gesprächspartner*innen und dem hamburgischen Substandard kann mithilfe der Sprachbewertungen näher beleuchtet werden: Neutrale Bewertungen wie bei den Merkmalspaaren „humorvoll - ernst“ und ,vornehm - grob“ betonen einerseits die Konzeptualisierung des Hamburgischen als Sprache des Alltags. Andererseits weisen positive Zuschreibungen und Kommentare wie bei den emotiven Eigenschaftspaaren ,gemütlich - ungemütlich“, ,freundlich - unfreundlich“ und ,sympathisch - unsympathisch“ darauf hin, dass der Regiolekt mit den Selbstkonzepten der Gesprächspartner*innen verknüpft wird und damit gleichermaßen Identität stiftet.

Die kommenden Analysen im Rahmen des Dissertationsprojekts „Regionale Sprache als Identitätsmarker. Zur Konstruktion sozialer Bedeutung bei Hamburger Fußballfans" werden durch Untersuchungen objektiver Sprachdaten (Anschauen eines Fußballspiels) ergänzt, um den Zusammenhang zwischen Spracheinstellungen und Sprachgebrauch näher zu beleuchten. Außerdem wird der Fokus auf die Teilidentität als Fußballfan gerichtet, indem die (außer-) sprachlichen Mittel und Strategien miteinbezogen werden, die die Selbstdarstellung sowie die Abgrenzung zum rivalisierenden Fanlager betreffen.

\section{Korpus}

HSV Fußball AG. https://de-de.facebook.com/HSV/ [Statusmeldungen zw. d. 08.04.2017 und

07.03.2018, zuletzt abgerufen zw. d. 15.05.2017 und 22.03.2018].

HSV Fußball AG. www.hsv.de/de/fans/offizielle-fanclubs/unsere-fanclubs/ [29.08.2018]. 
Fanclubsprecherrat St. Pauli. www.fanclubsprecherrat.de/fanclubs-2/ [29.08.2018].

\section{Literaturverzeichnis}

Agha, Asif (2005): „Voice, Footing, Enregisterment“. Journal of Linguistic Anthropology 15/1: $38-59$.

Allport, Gordon (1935): „Attitudes“. In: Murchison, Carl (ed.): A Handbook of Social Psychology. Worcester, Mass., Clark Univ. Press: 798-844.

Arendt, Birte (2010): Niederdeutschdiskurse. Spracheinstellungen im Kontext von Laien, Printmedien und Politik. Berlin: Erich Schmidt. (= Philologische Studien und Quellen, Heft 224).

Bamberg, Michael G. W. (1997): „Positioning Between Structure and Performance“. Journal of Narrative and Life History 7/1-4: 335-342.

Bergler, Reinhold/Six, Bernd (1972): „Stereotype und Vorurteile“. In: Graumann, Carl Friedrich (ed.): Sozialpsychologie. 2. Halbband: Forschungsbereiche. Göttingen, Verlag für Psychologie Dr. C. J. Hogrefe: 1371-1432. (=Handbuch der Psychologie in 12 Bänden 7/2)

Blotevogel, Hans Heinrich (2001): „Regionalbewusstsein und Landesidentität am Beispiel von Nordrhein-Westfalen“. In: Institut für Geographie Universität Duisburg. (ed.): Diskussionspapier 2/2001: 1-17. https://duepublico.uni-duisburg-essen.de/servlets/DerivateServlet/Derivate-5198/blotevogel2.pdf [11.10.2018].

Bohner, Gerd (2002): „Einstellungen“. In: Stroebe, Wolfgang/Jonas, Klaus/Hewstone, Miles (eds.): Sozialpsychologie. Eine Einführung. 4., überarbeitete und erweiterte Auflage, Berlin etc., Springer: $265-315$.

Bücker, Jörg (2015): „Selbstbeantwortete Fragen in der Mündlichkeit - ein Fall für die Konstruktionsgrammatik?“،. In: Bücker, Jörg/Günthner, Susanne/Imo, Wolfgang (eds.): Konstruktionsgrammatik V. Konstruktionen im Spannungsfeld von sequenziellen Mustern, kommunikativen Gattungen und Textsorten. Tübingen, Stauffenburg: 219-246.

Burkhardt, Armin (2006): Wörterbuch der Fußballsprache. Göttingen: Die Werkstatt.

Davies, Bronwyn/Harré, Rom (1990): „Positioning: The discursive production of selves“. Journal of the Theory of Social Behavior 20/1: 43-63.

Eagly, Alice H./Chaiken, Shelly (1993): The Psychology of Attitudes. Fort Worth etc.: Harcourt Brace Jovanovich.

Eagly, Alice H./Chaiken, Shelly (1998): „Attitude structure and function“. In: Gilbert, Daniel T./Fiske, Susan T./Lindzey, Gardner (eds.): The Handbook of Social Psychology. 4th ed./Vol. 1,. New York, McGraw-Hill: 269-322.

Eckert, Penelope (2008): „Variation and the indexical field“. Journal of Sociolinguistics 12/4: 453-476.

Eckert, Penelope (2012): „Three Waves of Variation Study: The Emergence of Meaning in the Study of Sociolinguistic Variation“. Annual Review of Anthropology 41: 87-100.

Ehlich, Konrad/Rehbein, Jochen (1976): „Halbinterpretative Arbeitstranskriptionen (HIAT)“. Linguistische Berichte 45: 21-41.

Elmentaler, Michael/Niebuhr, Oliver (2017): „Platt - Missingsch - Petuh: Enregistermentprozesse zwischen Hamburg und Flensburg“". In: Anderwald, Lieselotte/Hoekstra, Jarich (eds.): Enregisterment. Zur sozialen Bedeutung sprachlicher Variation. Frankfurt a. M., Lang: 107-141. (= Kieler Forschungen zur Sprachwissenschaft 8). 
Gehrmann, Siegfried (1994): „Football and identity in the Ruhr: the case of Schalke 04“. In: Giulianotti, Richard/Williams, John (eds.): Game Without Frontiers. Football, identity and modernity. Aldershot/Hants, Arena: 185-205. (= Popular Cultural Studies 5).

Gleber, Peter (1994): „Region und Identität: eine allgemeine Einführung“. In: Bossong, Georg et al. (eds.): Westeuropäische Regionen und ihre Identität. Beiträge aus interdisziplinärer Sicht. Mannheim, Palatium-Verlag im J \& J Verlag: 2-12. (= Mannheimer Historische Forschungen 4).

Grau, Andreas et al. (2016): „Football Fans in Germany: A Latent Class Analysis Typology“. Journal of Sporting Cultures and Identity 7/1: 19-31.

Grau, Andreas/Winands, Martin (2017): „Herausforderungen quantitativer und qualitativer Forschung in (Jugend-)Kulturen und Szenen. Das Beispiel der Fußballfanforschung“. In: Grau, Andreas/Heyde, Judith von der/Kotthaus, Jochem/Schmidt, Holger/Winands, Martin (eds.): Sozialwissenschaftliche Perspektiven in der Fußballfanforschung. Weinheim, Beltz Juventa: 56-74. (= Sportfans im Blickpunkt sozialwissenschaftlicher Forschung 1).

Haddock, Geoffrey/Maio, Gregory R. (2014): „Einstellungen“. In: Stroebe, Wolfgang/Jonas, Klaus/Hewstone, Miles (eds.): Sozialpsychologie. 6., vollständig überarbeitete Auflage, Berlin/Heidelberg, Springer: 197-229.

Heitmeyer, Wilhelm/Peter, Jörg-Ingo (1988): Jugendliche Fußballfans. Soziale und politische Orientierungen, Gesellungsformen, Gewalt. Weinheim/München: Juventa.

Herrmann, Hans Ulrich (1977): Die Fußballfans. Untersuchungen zum Zuschauersport. Schorndorf: Karl Hofmann. (= Beiträge zur Lehre und Forschung im Sport 60).

Hettler, Yvonne (2013): „,Nach Müllers geht man nicht!‘ Zur Salienz und Bewertung morpho-syntaktischer Phänomene in Bremen und Hamburg“. In: Hettler, Yvonne et al. (eds.): Variation, Wandel, Wissen. Studien zum Hochdeutschen und Niederdeutschen. Frankfurt a. M., Lang: 161-183. (= Sprache in der Gesellschaft 32).

Hettler, Yvonne (2016): ,,Die Bremer sprechen natürlich immer dieses ,e‘ so komisch. 'Laienlinguistische Selbst- und Fremdwahrnehmung in Bremen und Hamburg“. In: Bieberstedt, Andreas/Ruge, Jürgen/Schröder, Ingrid (eds.): Hamburgisch. Struktur, Gebrauch, Wahrnehmung der Regionalsprache im urbanen Raum. Frankfurt a. M. etc., Lang: 171-213. (= Sprache in der Gesellschaft 34).

Hettler, Yvonne (2018): Salienz, Bewertung und Realisierung regionaler Sprachmerkmale in Bremen und Hamburg. Hildesheim: Olms. (= Deutsche Dialektgeographie 119).

Heyde, Judith von der/Kotthaus, Jochem/Weitzel, Gerrit (2018): „,Wo Fußball intensiver gelebt wird“. Das Narrativ des ,Arbeitervereins“ Borussia Dortmund“. In: Denkler, Markus/Hartmann, Dietrich/Menge, Heinz H. (eds.): Dortmund - sprachliche Vielfalt in der Stadt. Wien/Köln/Weimar, Böhlau: 56-74. (=Niederdeutsche Studien 59).

Hundt, Markus/Palliwoda, Nicole/Schröder, Saskia (2017): Der deutsche Sprachraum aus der Sicht linguistischer Laien. Ergebnisse des Kieler DFG-Projektes. Berlin/Boston: de Gruyter. Johnstone, Barbara/Andrus, Jennifer/Danielson, Andrew E. (2006): „Mobility, Indexicality, and the Enregisterment of 'Pittsburghese'". Journal of English Linguistics 34/2: 77-104.

Johnstone, Barbara (2010): „Locating Language in Identity”. In: Llamas, Carmen/Watt, Dominic (eds.): Language and Identities. Edinburgh, Edinburgh Univ. Press: 29-36.

Jürgens, Carolin (2015a): Niederdeutsch im Wandel. Sprachgebrauchswandel und Sprachwahrnehmung in Hamburg. Hildesheim: Olms. (= Deutsche Dialektgeographie 119). 
Jürgens, Carolin (2015b): „Hamburgisch, Missingsch, Barmbek Basch. Die Wahrnehmung eines regionalen Substandards durch linguistische Laien in Hamburg“. In: Langhanke, Robert (ed.): Sprache, Literatur, Raum. Festgabe für Willy Diercks. Bielefeld, Verlag für Regionalgeschichte: 182-204.

Jürgens, Carolin (2016): „Regionale Identität per Einkaufstüte. Eine Fallstudie zum Enregisterment des Niederdeutschen in Hamburg“. In: Bieberstedt, Andreas/Ruge, Jürgen/Schröder, Ingrid (eds.): Hamburgisch. Struktur, Gebrauch, Wahrnehmung der Regionalsprache im urbanen Raum. Frankfurt a. M. etc., Lang: 307-343. (= Sprache in der Gesellschaft 34).

Jürgens, Carolin/Schröder, Ingrid (2016): „Sprachstereotype und ihre Realisierungen im Gespräch am Beispiel des Niederdeutschen“. In: Bieberstedt, Andreas/Ruge, Jürgen/Schröder, Ingrid (eds.): Hamburgisch. Struktur, Gebrauch, Wahrnehmung der Regionalsprache im urbanen Raum. Frankfurt a. M., Lang: 345-385. (= Sprache in der Gesellschaft 34).

Katz, Daniel (1960): The functional approach to the study of attitudes. Public Opinion Quarterly 24: 163-204.

König, Katharina (2014): Spracheinstellungen und Identitätskonstruktion. Eine gesprächsanalytische Untersuchung sprachbiographischer Interviews mit Deutsch-Vietnamesen. Berlin: de Gruyter. (=Empirische Linguistik / Empirical Linguistics 2).

Kotthaus, Jochem (2017): „Ultras als Szene. Methodologische Überlegungen zu einer Konzeptualisierung der Ultrabewegung als posttraditionale Vergemeinschaftung“. In: Grau, Andreas/Heyde, Judith von der/Kotthaus, Jochem/Schmidt, Holger/Winands, Martin (eds.): Sozialwissenschaftliche Perspektiven in der Fußballfanforschung. Weinheim, Beltz Juventa: 91-111. (= Sportfans im Blickpunkt sozialwissenschaftlicher Forschung 1).

Krappmann, Lothar (2004): „Identität/Identity“. In: Ammon, Ulrich/Dittmar, Norbert/Mattheier, Klaus J./Trudhill, Peter (eds.): Sociolinguistics/Soziolinguistik: An international handbook of the science of language and society/Ein internationales Handbuch zur Wissenschaft von Sprache und Gesellschaft. Berlin etc., de Gruyter: 405-412. (= Handbücher zur Sprachund Kommunikationswissenschaft / Handbooks of Linguistics and Communication Science 3/1).

Kresić, Marijana (2007): „Sprache der Identität“. In: Beiträge des Projekts „Signs of Identity“: 1-23. www.signsofidentity.de/fileadmin/pdf/Sprache_der_Identitaet_Beitrag_Kresic_ 8.6.07.pdf [11.10.2018].

Labov, William (1972): Sociolinguistic Patterns. Philadelphia: Univ. of Pennsylvania Press. (= Conduct and Communication No. 4).

Lasagabaster, David (2004): „Attitude/Einstellung“. In: Ammon, Ulrich/Dittmar, Norbert/Mattheier, Klaus J./Trudhill, Peter (eds.): Sociolinguistics/Soziolinguistik: An international handbook of the science of language and society/Ein internationales Handbuch zur Wissenschaft von Sprache und Gesellschaft. Berlin etc., de Gruyter: 399-405. (= Handbücher zur Sprach- und Kommunikationswissenschaft / Handbooks of Linguistics and Communication Science 3/1)

Lenhard, Michael (2002): Vereinsfussball und Identifikation in Deutschland. Phänomen zwischen Tradition und Postmoderne. Hamburg: Dr. Kovač. (= Schriften zur Sportwissenschaft 32).

Leuenberger, Petra (2000): „Ortsloyalität und Variationsverhalten.“ In: Häcki Buhofer, Annelies (ed.): Vom Umgang mit sprachlicher Variation. Soziolinguistik, Dialektologie, 
Methoden und Wissenschaftsgeschichte. Festschrift für Heinrich Löffler zum 60. Geburtstag. Tübingen/Basel, A. Franke: 159-172. (=Baseler Studien zur deutschen Sprache und Literatur 80).

Lilli, Waldemar (1994): „Sozialpsychologische Implikationen Regionaler Identität“. In: Bossong, Georg et al. (eds.): Westeuropäische Regionen und ihre Identität. Beiträge aus interdisziplinärer Sicht. Mannheim, Palatium-Verlag im J \& J Verlag: 85-98. (= Mannheimer Historische Forschungen 4).

Löffler, Heinrich (1998): „Dialekt und regionale Identität. Neue Aufgaben für die Dialektforschung“. In: Ernst, Peter/Patocka, Franz (eds.): Deutsche Sprache in Raum und Zeit. Festschrift für Peter Wiesinger zum 60. Geburtstag. Wien, Edition Praesens: 71-85.

Lucius-Hoene, Gabriele/Deppermann, Arnulf (2002): Rekonstruktion narrativer Identität: Ein Arbeitsbuch zur Analyse narrativer Interviews. Opladen: Leske + Budrich.

Lucius-Hoene, Gabriele/Deppermann, Arnulf (2004): „Narrative Identität und Positionierung“. Gesprächsforschung - Online-Zeitschrift zur verbalen Interaktion 5: 166-183. http://www.gespraechsforschung-online.de/heft2004/ga-lucius.pdf [11.10.2018].

Mayring, Philipp (2015): Qualitative Inhaltsanalyse: Grundlagen und Techniken. 12., überarbeitete Auflage, Weinheim/Basel: Beltz.

Neuland, Eva (1993): „Sprachgefühl, Spracheinstellungen, Sprachbewusstsein. Zur Relevanz ,subjektiver Faktoren' für Sprachvariation und Sprachwandel“". In: Mattheier, Klaus J. et al. (eds.): Vielfalt des Deutschen. Festschrift für Werner Besch. Frankfurt a. M. etc., Lang: 723747.

Neumann, Lara/Schröder, Ingrid (2017a): „Identitätskonstruktionen in sprachbiographischen Interviews. Analysen zur Funktion des Niederdeutschen in Hamburg“. In: Schröder, Ingrid/Jürgens, Carolin (eds.): Sprachliche Variation in autobiographischen Interviews. Theoretische und methodische Zugänge. Frankfurt a. M. etc., Lang: 225-242. (= Sprache in der Gesellschaft 35).

Neumann, Lara/Schröder, Ingrid (2017b): „Zur Bewertung von Niederdeutsch und lokalem Substandard in Hamburg“. Linguistik Online 85/6: 227-255.

Preston, Dennis R. (2010): „Perceptual Dialectology in the 21st Century“. In: Anders, Ada Christina/Hundt, Markus/Lasch, Alexander (eds.): Perceptual Dialectology. Neue Wege der Dialektologie. Berlin/New York, de Gruyter: 1-29. (= Linguistik - Impulse \& Tendenzen 38).

Rosa, Hartmut (2007): „Identität“. In: Straub, Jürgen/Weidemann, Arne/Weidemann, Doris (eds.): Handbuch interkulturelle Kommunikation und Kompetenz. Grundbegriffe - Theorien - Anwendungsfelder. Stuttgart/Weimar, J.B. Metzler: 47-56.

Rosenberg, Milton J./Hovland, Carl I. (1960): „Cognitive, Affective and Behavioral Components of Attitudes“. In: Rosenberg, Milton J./Hovland, Carl I. (eds.): Attitude Organization and Change: An Analysis of Consistency among Attitude Components. New Haven, Conn., Yale Univ. Press: 1-14.

Schmidt, Jürgen Erich/Herrgen, Joachim (2011): Sprachdynamik. Eine Einführung in die moderne Regionalsprachenforschung. Berlin: Erich Schmidt. (= Grundlagen der Germanistik 49).

Schröder, Ingrid (2010): „Plattdeutsch in Hamburg. Sprachwahl als Mittel zur Konstruktion lokaler Identität?“ In: Müns, Wolfgang (ed.): Man mag sik kehrn un kanten, as man will, 
noch jümmer is der'n Eck, wo man ni wen is. 100. Jahrgang der Zeitschrift "Quickborn “. Festschrift. Hamburg, Quickborn-Verlag: 585-601. (= Quickborn-Bücher 100/101).

Schröder, Ingrid (2015): „Von der Dialektologie zur Regionalsprachenforschung - eine norddeutsche Perspektivierung“. In: Elmentaler, Michael/Hundt, Markus/Schmidt, Jürgen Erich (eds.): Deutsche Dialekte. Konzepte, Probleme, Handlungsfelder. Akten des 4. Kongresses der Internationalen Gesellschaft für Dialektologie des Deutschen. Stuttgart, Steiner: 25- 57. (= Zeitschrift für Dialektologie und Linguistik, Beihefte 158).

Schröder, Ingrid/Jürgens, Carolin (2017): „Einstellungen gegenüber regionalen Sprachformen in der Großstadt: Niederdeutsch in Hamburg. Eine Projektskizze“. In: Schröder, Ingrid/Jürgens, Carolin (eds.): Sprachliche Variation in autobiographischen Interviews. Theoretische und methodische Zugänge. Frankfurt a. M. etc., Lang: 11-46. (= Sprache in der Gesellschaft 35).

Schröder, Ingrid (2019): „Sprachbiographie und Spracheinstellung. Niederdeutsch als Mittel der Identitätsstiftung in der Großstadt?“ In: Eichinger, Ludwig M./Plewnia, Albrecht (eds.): Neues vom heutigen Deutsch. Empirisch - methodisch - theoretisch. Berlin/Boston, de Gruyter: 99-120. (= Jahrbuch des Instituts für Deutsche Sprache 2018).

Silverstein, Michael (2003): „Indexical order and the dialectics of sociolinguistic life“. Language and Communication 23/3-4: 193-229.

Spitzmüller, Jürgen (2013): „Metapragmatik, Indexikalität, soziale Registrierung. Zur diskursiven Konstruktion sprachideologischer Positionen“. Zeitschrift für Diskursforschung 3: 263-287.

Straub, Jürgen (2011): „Identität“. In: Jaeger, Friedrich/Liebsch, Burkhard (eds.): Handbuch der Kulturwissenschaften. Grundlagen und Schlüsselbegriffe. Bd. 1, Stuttgart/Weimar, J.B. Metzler: 277-303.

Tajfel, Henri (1981): Human Groups and Social Categories. Studies in Social Psychology. Cambridge: Cambridge Univ. Press.

Thim-Mabrey, Christiane (2003): „Sprachidentität - Identität durch Sprache. Ein Problemaufriss aus sprachwissenschaftlicher Sicht“. In: Janich, Nina/Thim-Mabrey, Christiane (eds.): Sprachidentität - Identität durch Sprache. Tübingen, Narr: 1-18.

Tophinke, Doris/Ziegler, Evelyn (2006): „,Aber bitte im Kontext!' Neue Perspektiven der dialektologischen Einstellungsforschung“. In: Voeste, Anja/Gessinger, Joachim (eds.): Dialekt im Wandel. Perspektiven einer neuen Dialektologie. Duisburg etc., Univ.-Verlag RheinRuhr: 205-224. (= Osnabrücker Beiträge zur Sprachtheorie (OBST) 71).

Verein Jugend und Sport e.V. (1996): Lokalrivalität. Ihr sollt sie ja nicht lieben, aber... Dokumentation der Begleitmaßnahmen um das Derby HSV - FC St. Pauli am 24. November 1995. Hamburg: Selbstverlag.

Winands, Martin/Grau, Andreas/Zick, Andreas (2017): „Sources of identity and community among highly identified football fans in Germany. An empirical categorization of differentiation processes“. Soccer \& Society: 1-16.

Zanna, Mark P./Rempel, John K. (1988): „Attitudes: A new look at an old concept“. In: BarTal, Daniel/Kruglanski, Arie W. (eds.): The Social Psychology of Knowledge. Cambridge, Cambridge Univ. Press: 315-334. 